\title{
Amino acids in acute-on-chronic liver failure: Another piece of the puzzle?
}

\author{
Minneke J. Coenraad ${ }^{1, *}$, Florent Artru ${ }^{2}$ \\ ${ }^{1}$ Department of Gastroenterology and Hepatology, Leiden University Medical Center, Leiden, The Netherlands; ${ }^{2}$ Division of Gastroenterology \\ and Hepatology, University Hospital and University of Lausanne, Lausanne, Switzerland
}

See Article, pages 1117-1131

In acute-on-chronic liver failure (ACLF), there is an unmet need for biomarkers to assess prognosis, treatment response and for the identification of leads for targeted therapy. In order to meet these needs, understanding the pathophysiology and sequence of events is required. In particular, the causal and temporal relationships between inflammation and organ dysfunctions need to be elucidated.

In this issue of Journal of Hepatology, Zaccherini and Aguilar et al. ${ }^{1}$ present a comprehensive study, aiming to address the role of amino acids (AAs) in systemic inflammation and organ failures in ACLF. These researchers recently showed that patients with ACLF have a distinctive metabolic fingerprint, characterized by proteolysis, lipolysis and extramitochondrial glucose metabolism, with enhanced aerobic glycolysis and increased levels of blood metabolites related to the pentose phosphate pathway, with subsequent depressed mitochondrial energy production. ${ }^{2}$ In parallel, they reported enhanced extramitochondrial AA catabolism $^{2,3}$ and hypothesized that the observed altered glucose metabolism may be associated with changes in AA metabolism. Overall, the altered metabolism of both glucose and AA could contribute to immune activation and organ failure in ACLF.

The authors are to be congratulated for their comprehensive and original study and for providing the background information on key metabolic processes, to put into context the physiological consequences of observed metabolic alterations. The authors used a recently proposed and original approach - weighted gene co-expression network analysis - to analyse the whole metabolomic data set. This data mining method allowed them to study biological networks by defining modules in high-dimensional data sets, based on correlations between molecules. All metabolites captured in the same module are therefore considered to be driven by the same metabolic purpose.

The authors reported a profound metabolic reprogramming with a significant metabolite accumulation in patients with ACLF. They

\footnotetext{
Keywords: Multiorgan failure; Small-molecules; Biomass; Immunity; Anabolic metabolism; Dormancy; Hyperammonemia.

Received 28 December 2020; accepted 7 January 2021; available online 20 February 2021

DOI of original article: https://doi.org/10.1016/j.jhep.2020.11.035.

* Corresponding author. Address: Department of Gastroenterology and Hepatology, C4-P-014, Leiden University Medical Center, Albinusdreef 2, Leiden, ZA 2333, The Netherlands. Tel.: +31-71-5263507/ +31-71-5265217, fax: +31-71-5248115.

E-mail address: m.j.coenraad@lumc.nl (M.J. Coenraad).

https://doi.org/10.1016/j.jhep.2021.01.009
}

hypothesized, based on what is observed in septic non-cirrhotic patients, that AAs fuel anabolic programs, including protein and nucleotide synthesis, required for the intense systemic inflammatory response and for energy supply to peripheral organs. Interestingly, the observed metabolic reprogramming of AAs may functionally impact on components of the inflammatory response. To illustrate this, Korf et al. ${ }^{4}$ showed that monocytes from patients with ACLF show an immunosuppressive signature with defective phagocytic and inflammatory functions. Monocyte dysfunction could be restored by metabolic rewiring, e.g. by feeding glutamine into the tricarboxylic acid cycle within monocytes using a pharmacological inhibitor of glutamine synthetase. In this context, the new metabolomics data provided by Zaccherini and Aguilar et al. may provide new insights on metabolite use and may aid in tracking the consequences of therapeutic interventions. The authors have confirmed with dedicated analyses that blood metabolite accumulation in ACLF was not solely the result of defective renal excretion. Indeed, only 2 eigenmetabolites were upregulated in patients with single kidney failure compared to those without kidney failure.

The impact of other potentially relevant factors, such as bacterial origin of metabolic products and the interaction with the host, nutritional status or the circadian rhythm could not be assessed. The human gut microbiome, including the interplay between the host and the gut, reflected by changes in the metabolome, will be extensively studied in patients with decompensated cirrhosis and ACLF in the Horizon 2020-funded MICROB-PREDICT project (https://microb-predict.eu/). Regarding circadian rhythm, samples were collected at time of enrolment in the CANONIC study, while time of sampling was not standardized. Under highly controlled conditions, healthy individuals show significant $24 \mathrm{~h}$ variations in several metabolites, including the amino acids methionine, tyrosine, proline, lysine, phenylalanine and leucine, with a mean ratio of oscillation range relative to the MESOR of $65 \%$, with the highest level of variation from mid- to late afternoon. ${ }^{5}$ The relevance of time-ofday variations, in the context of multiple other factors impacting on metabolic derangements, cannot be determined from the present study. In future metabolite-based studies, recognition of potential $24 \mathrm{~h}$ variation is warranted for correct interpretation of study results. In the present study, the lack of a control cohort of patients with conditions known to be associated with intense systemic inflammatory features (e.g. critically ill patients, septic patients) makes it difficult to assess the disease specificity of the 
authors' observation. Particularly because the authors rely on a hypothesis validated in sepsis under non-cirrhotic conditions, that the observed changes in blood metabolites aim to fuel the intense innate immune response. Some similarities in the analyses of blood metabolomics in the 2 groups may confirm this assumption (increased pyruvate, lactate, $\alpha$-ketoglutarate, oxaloacetate and acylcarnitines in patients with worst prognosis). Other common features are shared between patients with ACLF and critically ill and/or septic patients, e.g. mitochondrial dysfunction $^{6}$ which was recently reported to reduce ATPproducing fatty acid $\beta$-oxidation and subsequently oxidative phosphorylation in ACLF. ${ }^{2}$ Interestingly, a defect in autophagy, an anti-inflammatory process that may help preserve mitochondrial function, has been suggested by decreased blood levels of spermidine in the present paper. Defective autophagy is known to be associated with outcome in sepsis and critical illness.,

Another common feature between patients with ACLF and other groups of critically ill patients is the presence of skeletal muscle loss or sarcopenia, a condition commonly associated with poor outcome in different conditions. ${ }^{9-11}$ In cirrhotic patients, sarcopenia has been associated with development of ACLF. ${ }^{12}$ One of the main sources of AAs in intense anabolic and/or catabolic conditions is muscle proteolysis. In the present study, $43 \%$ of the metabolomics data set was AA-derived and the yellow module (gathering coregulated metabolites) was exclusively composed of AAs, underlining the magnitude of this process during ACLF. While in the 3 different conditions (critically ill, sepsis, cirrhosis), muscle proteolysis shares common pathways, e.g. systemic inflammation and myostatin-induced inhibition of muscle growth via the ubiquitin-proteasome pathway, ${ }^{13,14}$ the pivotal place of hyperammonemia as a mediator of the liver-muscle axis seems to be specific to patients with cirrhosis. In cirrhosis, hyperammonemia induces a cellular response that resembles some features of $\mathrm{AA}$ starvation $^{15}$ and is responsible for the increased expression of myostatin, leading to skeletal muscle loss. ${ }^{16,17}$ While ammonialowering therapy has been suggested to reverse sarcopenia by restoring proteostasis in hyperammonemic animal models, ${ }^{18}$ it is interesting to notice that supplementation with L-leucine, a branched chain amino acid, allowed the loss of inhibition in protein synthesis. In the paper by Zaccherini and Aguilar et al., the authors suggest that the urea cycle, usually enabling ammonia detoxification, could be deregulated while its key molecules are being diverted to fuel nucleotide synthesis. These data suggest that targeted nutritional support is of potential benefit to patients with cirrhosis and ACLF. Surprisingly, the authors also reported a probable defect or inhibition of ketone body production from AAs, with the absence of the canonical ketone body $\beta$-hydroxybutyrate in their metabolomic data set. Indeed, ketogenesis is observed in sepsis and its intensity is associated with outcome in animal models and humans. ${ }^{19,20}$ Moreover, increased availability of ketone bodies has recently been suggested to protect against sepsisinduced muscle wasting, that could be linked with the increase in sarcopenia observed in ACLF. ${ }^{19}$ The absence of ketogenesis could therefore be a key feature of ACLF that should be studied.

This multicentre study has multiple strengths: the overall cohort of patients is large and well described, with detailed characteristics at admission and during follow-up and uniform sample collection at hospital admission. Nevertheless, the study is descriptive and associative, and does not contain any mechanistic investigations, as properly discussed in the manuscript. Conclusions should therefore be interpreted cautiously.
The present study provides new evidence regarding massive metabolic reprogramming in ACLF, and as such, it may serve as a valuable resource paper for future basic investigations aimed at increased understanding of pathophysiological mechanisms of ACLF and biomarker research. It would be interesting to study the temporal resolution of observed metabolic derangements in relation to the disease trajectory by serial sampling in patients in order to elucidate the antecedence of metabolic reprogramming before development of ACLF or death. In fact, the CANONIC study provides a unique opportunity to evaluate intra-individual sequential changes in AA metabolism from patients with AD progressing to ACLF, providing the ultimate proof of ACLF-specific AA metabolic pathways. In addition, studies are warranted to challenge the mechanisms proposed by the authors, such as the competition which may occur if metabolic costs of acute systemic inflammatory activation exceed the supply, inducing an energyconserving hypometabolic state (dormancy program) in peripheral organs, and the tissue specificity of the observed metabolic derangements. Studying tissue specificity is complicated because of ethical constraints on taking tissue samples from different organs in humans. As an alternative, representative animal models or cell models could be applied, although they may not recapitulate all relevant aspects of the disease. In conclusion, the observed metabolic reprogramming in ACLF warrants future mechanistic study.

\section{Financial support}

The authors received no financial support to produce this manuscript.

\section{Conflict of interest}

The authors declare no conflicts of interest that pertain to this work.

Please refer to the accompanying ICMJE disclosure forms for further details.

\section{Authors' contributions}

$\mathrm{MC}$ and FA contributed equally to the concept, writing the draft and approving the final version of the manuscript.

\section{Supplementary data}

Supplementary data to this article can be found online at https:// doi.org/10.1016/j.jhep.2021.01.009.

\section{References}

Author names in bold designate shared co-first authorship

[1] Zaccherini G, Aguilar F, Caraceni P, Clària J, Lozano JJ, Fenaille F, et al. Assessing the role of amino acids in systemic inflammation and organ failure in patients with ACLF. J Hepatol 2021;74:1117-1131.

[2] Moreau R, Clària J, Aguilar F, Fenaille F, Lozano JJ, Junot C, et al CANONIC study investigators of the EASL clif consortium; European foundation for the study of chronic liver failure (EF clif). Blood metabolomics uncovers inflammation-associated mitochondrial dysfunction as a potential mechanism underlying ACLF. J Hepatol 2020;72:688-701.

[3] Clària J, Moreau R, Fenaille F, Amorós A, Junot C, Gronbaek H, et al CANONIC study investigators of the EASL clif consortium, grifols chair and the European foundation for the study of chronic liver failure (EF clif). Orchestration of tryptophan-kynurenine pathway, acute decompensation, and acute-on-chronic liver failure in cirrhosis. Hepatology 2019;69:16861701. 
[4] Korf H, Du Plessis J, Van Pelt J, De Groote S, Cassiman D, Verbeke L, et al. Inhibition of glutamine synthetase in monocytes from patients with acute-on-chronic liver failure resuscitates their antibacterial and inflammatory capacity. Gut 2019;68:1872-1883.

[5] Revell V, Mann A, Mäntele S, Otway DT, Johnston JD, Thumser AE, et al. Identification of human plasma metabolites exhibiting time-of-day variation using an untargeted liquid chromatography-mass spectrometry metabolomic approach. Chronobiol Int 2012;29:868-881.

[6] Brealey D, Brand M, Hargreaves I, Heales S, Land J, Smolenski R, et al. Association between mitochondrial dysfunction and severity and outcome of septic shock. Lancet 2002;360:219-223.

[7] Takahashi W, Watanabe E, Fujimura L, Watanabe-Takano H, Yoshidome $H$, Swanson PE, et al. Kinetics and protective role of autophagy in a mouse cecal ligation and puncture-induced sepsis. Crit Care 2013;17:R160.

[8] Tardif N, Polia F, Tjäder I, Gustafsson T, Rooyackers O. Autophagy flux in critical illness, a translational approach. Sci Rep 2019;9:10762.

[9] Ebadi M, Bhanji RA, Mazurak VC, Montano-Loza AJ. Sarcopenia in cirrhosis: from pathogenesis to interventions. J Gastroenterol 2019;54:845-859.

[10] Schefold JC, Wollersheim T, Grunow JJ, Luedi MM, Z'Graggen WJ, WeberCarstens SJ. Muscular weakness and muscle wasting in the critically ill. J Cachexia Sarcopenia Muscle 2020;11:1399-1412.

[11] Schefold JC, Bierbrauer J, Weber-Carstens S. Intensive care unit-acquired weakness (ICUAW) and muscle wasting in critically ill patients with severe sepsis and septic shock. J Cachexia Sarcopenia Muscle 2010;1:147157.

[12] Praktiknjo M, Book M, Luetkens J, Pohlmann A, Meyer C, Thomas D, et al. Fat-free muscle mass in magnetic resonance imaging predicts acute-on- chronic liver failure and survival in decompensated cirrhosis. Hepatology 2018;67:1014-1026.

[13] Zimmers TA, Davies MV, Koniaris LG, Haynes P, Esquela AF, Tomkinson KN, et al. Induction of cachexia in mice by systemically administered myostatin. Science 2002;296:1486-1488.

[14] McFarlane C, Plummer E, Thomas M, Hennebry A, Ashby M, Ling N, et al. Myostatin induces cachexia by activating the ubiquitin proteolytic system through an NF-kappaB-independent, FoxO1-dependent mechanism. J Cell Physiol 2006;209:501-514.

[15] Davuluri G, Krokowski D, Guan B-J, Kumar A, Thapaliya S, Singh D, et al. Metabolic adaptation of skeletal muscle to hyperammonemia drives the beneficial effects of 1-leucine in cirrhosis. J Hepatol 2016;65:929-937.

[16] Dasarathy S, McCullough AJ, Muc S, Schneyer A, Bennett CD, Dodig M, et al. Sarcopenia associated with portosystemic shunting is reversed by follistatin. J Hepatol 2011;54:915-921.

[17] Qiu J, Thapaliya S, Runkana A, Yang Y, Tsien C, Mohan ML, et al. Hyperammonemia in cirrhosis induces transcriptional regulation of myostatin by an NF-кB-mediated mechanism. Proc Natl Acad Sci U S A 2013;110:18162-18167.

[18] Kumar A, Davuluri G, Silva RNE, Engelen MPKJ, Ten Have GAM, Prayson R, et al. Ammonia lowering reverses sarcopenia of cirrhosis by restoring skeletal muscle proteostasis. Hepatology 2017;65:2045-2058.

[19] Goossens C, Weckx R, Derde S, Dufour T, Vander Perre S, Pauwels L, et al. Adipose tissue protects against sepsis-induced muscle weakness in mice: from lipolysis to ketones. Crit Care 2019;23:236.

[20] Langley RJ, Tsalik EL, van Velkinburgh JC, Glickman SW, Rice BJ, Wang C, et al. An integrated clinico-metabolomic model improves prediction of death in sepsis. Sci Transl Med 2013;5:195ra95. 